\title{
Simulation and Optimization of Nonperiodic Plasmonic Nano-Particles
}

\author{
Majid Akhlaghi*, Farzin Emami, Mokhtar Sha Sadeghi, and Mohammad Yazdanypoor \\ Department of Opto Electronic, Shiraz University of Technology, Shiraz, Iran
}

(Received November 6, 2013 : revised December 26, 2013 : accepted January 21, 2014)

\begin{abstract}
A binary-coupled dipole approximation (BCDA) is described for designing metal nanoparticles with nonperiodic structures in one, two, and three dimensions. This method can be used to simulate the variation of near- and far-field properties through the interactions of metal nanoparticles. An advantage of this method is in its combination with the binary particle swarm optimization (BPSO) algorithm to find the best array of nanoparticles from all possible arrays. The BPSO algorithm has been used to design an array of plasmonic nanospheres to achieve maximum absorption, scattering, and extinction coefficient spectra. In BPSO, a swarm consists of a matrix with binary entries controlling the presence (' 1 ') or the absence (' 0 ') of nanospheres in the array. This approach is useful in optical applications such as solar cells, biosensors, and plasmonic nanoantennae, and optical cloaking.
\end{abstract}

Keywords: Plasmonic nano particles, Optimization algorithm, Binary PSO

OCIS codes : (240.0240) Optics at surfaces; (240.6680) Surface plasmons; (070.0070) Fourier optics and signal processing

\section{INTRODUCTION}

Metal nanoparticles have broad applications in electronics, photonics, chemical sensing, and imaging [1-3]. Recently there has been a growing interest in plasmonic nanoparticles. They have strong effects on light in the visible and invisible regions of the photonic spectrum for applications such as Raman scattering [4], radiative rate enhancement [5], solar cells [6], and optical biosensors [7]. Since the optical properties of these particles depend on their sizes and shapes, one of the desired goals is to control the shapes of metal and semiconductor nanoparticles. Shape control has been successfully demonstrated for gold nanoparticles using nonionic surfactants, silver underpotential deposition, and nanoreplica molding [8-11]. Nanoreplica molding has been demonstrated as a low-cost method for manufacturing a variety of devices comprised of nano structured surfaces. Recently a plasmonic nanodome array was fabricated by the nanoreplica molding process [10]. In addition to the recent interest in shape control of nanoparticles, the optical properties of noble metal particles, with their intense colors, have fascinated scientists since the turn of this century. For example, spherical gold nanoparticles show a strong absorption band in the visible region of the electromagnetic spectrum at about $520 \mathrm{~nm}$ [12]. In addition to the shape, size, and material of nanoparticles, the properties of transmitted light strongly depend on the localized positions and gaps between nanoparticles. Plasmonic nanoparticles with periodic structure have been reported $[13,14]$. One of the most promising plasmonics nanoparticle platforms is studying the effect of deterministic aperiodic structure of nanoparticles on the properties of transmitted light [15]. Such structure, which is intermediate between a disordered system and a periodic one, enables unique control and manipulation of spatially localized plasmonic states over broadband frequency and angular spectra [16].

In structures with periodic plasmonic nanoparticles the characteristics of transmitted light can be calculated efficiently using the coupled-dipole approximation (CDA), originally developed by Purcell and PennyPacker [17] and improved by Draine [18]. In this paper, an alternative design method called the binary coupled dipole approximation (BCDA) is described. Utilizing this method, the effect of a nonperiodic structure of nanoparticles on light properties could be studied. A big advantage of this method is its application to optimization problems by utilizing binary evolutionary computational techniques, such as

\footnotetext{
*Corresponding author: m.akhlaghi@sutech.ac.ir

Color versions of one or more of the figures in this paper are available online.
} 
binary genetic algorithms and binary particle swarm optimization. Moreover, higher absorption, scattering, and extinction coefficients are obtained using BPSO and BCDA methods. These improvements can be helpful in optical applications such as solar cells, optical bio sensors, and plasmonic nanoantennae.

\section{METHODS}

\subsection{Binary Couple Dipole Approximation}

A schematic diagram of a three-dimensional array of arbitrarily shaped objects periodically arranged in the $\mathrm{x} \mathrm{y} \mathrm{z}$ plane is shown in Fig. 1(a). In the first time step the $x, y$, and $\mathrm{z}$ positions of each particle are calculated. To assign the position of each particle, it is assumed that there is a cube filled with nanoparticles equally distant from each other. The Matlab code for determining the positions of $\mathrm{N}$ particles is presented in Fig. 1(b). In this code, $\mathrm{r}$ is a particle's position vector, N1, N2, and N3 represent the odd number of particles in the $\mathrm{i}, \mathrm{j}$, and $\mathrm{k}$ directions, and $\mathrm{d}$ represents the distance between the particles. $\mathrm{B}(\mathrm{i}, \mathrm{j}, \mathrm{k})$ is a three-dimensional array of binary numbers. The values 1 and 0 in the $\mathrm{B}$ matrix refer respectively to the presence and absence of particles in the desired locations. Next, the incident electric field is simulated. The scattered light may be illuminated by any form of incident electric field, the simplest of which is a plane wave. To calculate the E-field of each dipole at $\mathrm{rj}$, the time harmonic component $-\mathrm{i} \omega \mathrm{t}$ of the E-field is left out. The Local field arising from incident light with polar $(\theta)$ and azimuthal $(\varphi)$ angles at

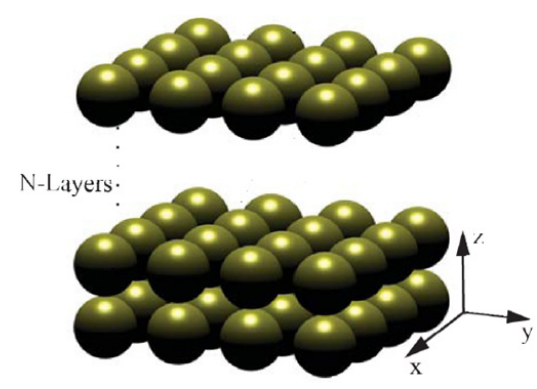

(a)

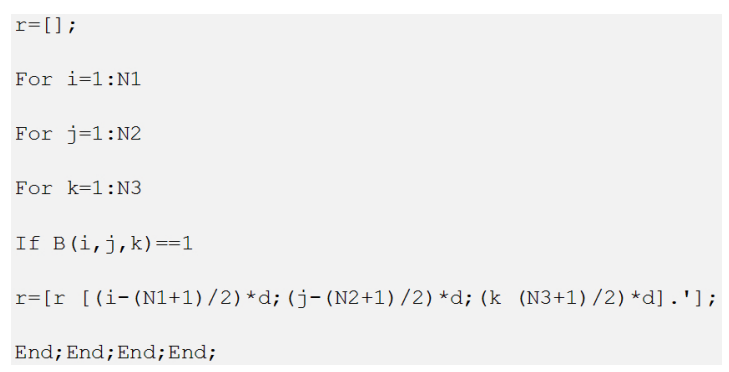

(b)

FIG. 1. (a) 3-dimensional array of nanoparticles. (b) Matlab code for determining the position of $\mathrm{N}$ number of nanoparticles. each particle is:

$$
\vec{E}_{\text {inc }}\left(\overrightarrow{r_{-i}}\right)=\vec{E}_{0} e^{i \vec{k} \cdot \vec{r}_{-i}}
$$

Where:

$$
\bar{k}=\frac{2 \pi}{\lambda} \hat{k}=\frac{2 \pi}{\lambda}[\sin (\theta) \cdot \cos (\varphi), \sin (\theta) \cdot \sin (\varphi), \cos (\theta)]
$$

For an incident field with p-polarization, the following can be written:

$$
E_{0}=\left[\sin \left(\theta-\frac{\pi}{2}\right) \cdot \cos (\varphi), \sin \left(\theta-\frac{\pi}{2}\right) \cdot \sin (\varphi), \cos \left(\theta-\frac{\pi}{2}\right)\right]
$$

and for an incident field with s-polarization:

$$
E_{0}=\left[\cos \left(\varphi+\frac{\pi}{2}\right), \sin \left(\varphi+\frac{\pi}{2}\right), 0\right]
$$

When the applied field is parallel to one of the principle axes, the polarizability $\alpha$ is [19]:

$$
\alpha=V \frac{\varepsilon-\varepsilon_{m}}{\varepsilon_{m}+L_{1}\left(\varepsilon-\varepsilon_{m}\right) F}
$$

where $\mathrm{V}$ is particle volume, $\varepsilon_{m}$ is the dielectric function of the surrounding medium, $\mathrm{L}$ is the shape factor, and
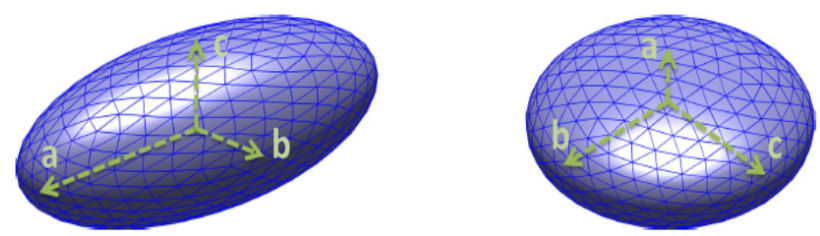

FIG. 2. Prolate (left, $\mathrm{a}>\mathrm{b}=\mathrm{c}$ ) and oblate (right, $\mathrm{a}<\mathrm{b}=\mathrm{c}$ ) ellipsoids.

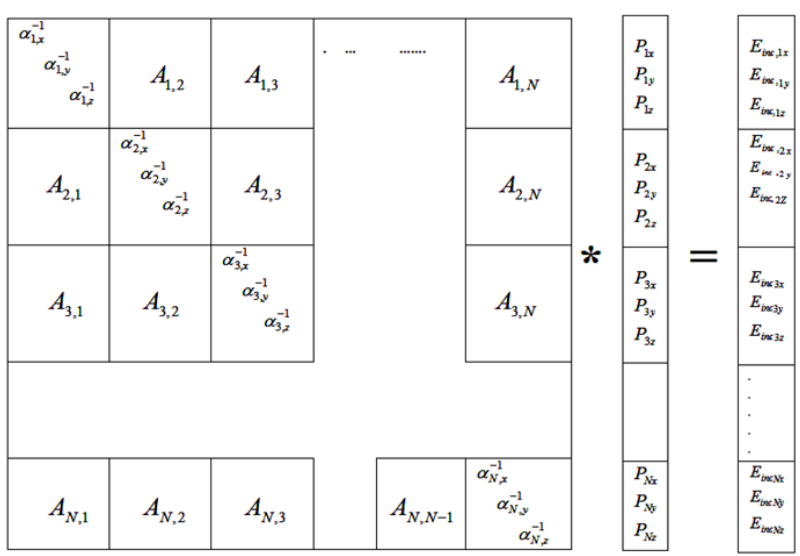

FIG. 3. A, P, Einc matrix from equation 10. 
$\mathrm{F}=1-(\mathrm{ka})-\frac{2}{3} i(k a)^{3}$. One advantage of the BCDA method is studying the effect of an ellipsoidal particle on light. For oblate and prolate spheroids $(\mathrm{b}=\mathrm{c})$, the following analytical expression can be given for $\mathrm{L} 1$ as a function of eccentricity e [19]:

$$
\begin{aligned}
& e^{2}=1-\frac{b^{2}}{a^{2}}, f^{2}=\frac{b^{2}}{a^{2}}-1 \\
& L_{1}=\frac{1+f^{2}}{f^{2}}\left[1-\frac{1}{f} \tan ^{-1}(f)\right] \quad \text { oblate }(a<b) \\
& L_{1}=\frac{1-e^{2}}{e^{2}}\left(-1+\frac{1}{2 e} \ln \frac{1+e}{1-e}\right) \quad \text { prolate }(a>b)
\end{aligned}
$$

where $a, b$, and $c$ are seminal excess of an ellipsoid $(a>b>$ c) and, for a sphere, $L_{1}=1 / 3$.

The dipole moment induced in a single particle by a local electric field is given by:

$$
\vec{P}_{-i}=\varepsilon_{0} \alpha_{i} \vec{E}_{l o c}\left(\vec{r}_{-}\right)
$$

Here, $\vec{P}_{i}$ is the induced dipole moment, $\alpha_{i}$ is the polarizability of the particle centered at $\vec{r}_{i}, E_{\text {loc }}$ is the local electric field, and $\varepsilon_{0}$ is the permittivity of free space. The local field arises from two sources: the first term is incident light, $\overrightarrow{E_{i n c}}\left(\vec{r}_{-}\right)=\vec{E}_{0} e^{i k r_{-} i}$, and the second term is superposition $\vec{E}_{\text {ret }}$ of the retarded fields from each of the other N-1 radiating dipoles in the array. Combining these terms leads to the local field as follows:

$$
\begin{aligned}
& \vec{E}_{i n c, i}=A_{i i} \overrightarrow{P_{-i}}+\sum_{j \neq i} A_{i j} \overrightarrow{P_{-i}} \\
& \left\{\begin{array}{l}
A_{i j}=\frac{\exp \left(i k \vec{r}_{i j}\right)}{\vec{r}_{i j}}\left[k^{2}\left(r_{i j} r_{i j}-I_{3 \times 3}\right)+\frac{i k \vec{r}_{i j}-1}{\vec{r}_{i j}^{2}}\left(3 r_{i j} r_{i j}-I_{3 \times 3}\right)\right], \quad j \neq i \\
A_{i i}=\frac{1}{\alpha_{i} \varepsilon_{0} I_{3 \times 3}}
\end{array}\right.
\end{aligned}
$$

Here, $\overrightarrow{r_{i j}}=\overrightarrow{r_{-}}-\overrightarrow{r_{j}}, r_{i j}=\left|r_{i j}\right|, \mathrm{k}$ is wave vector, and $\mathrm{A}_{\mathrm{ij}}$ are $3 \times 3$ matrices representing the interaction of two particles $\mathrm{i}$ and $\mathrm{j}$. Now, $3 \mathrm{~N}$ unknown dipole moments $\mathrm{Pj}$ should be solved for in the following exactly determined system of $3 \mathrm{~N}$ linear equations:

$$
\text { A.P }=E_{\text {inc }}
$$

where $A$ is a $3 \mathrm{~N} \times 3 \mathrm{~N}$ matrix containing $\mathrm{N} \_\mathrm{N}$ of $\mathrm{A}_{\mathrm{ij}} 3 \times 3$ tensors, where $\mathrm{N}$ is the number of dipoles and both $\mathrm{p}$ and
$\mathrm{E}_{\text {inc }}$ are $3 \mathrm{~N}$ vectors (i.e. each of $\mathrm{N}$ particles is represented by a 3-vector). These matrices are shown in Fig. 3. When this set of $3 \mathrm{~N}$ complex linear equations is solved $\left(A . P=E_{\text {inc }}\right)$, the $\mathrm{P}$ array of self-consistent dipole moments is obtained. The optical properties may be then calculated from this dipole array.

\subsection{Far-Field Properties of Light}

As explained in the previous section, optical absorption, scattering, and extinction can be directly calculated from the dipole array. The extinction and scattering coefficients are [12]:

$$
\begin{gathered}
Q_{e x t}=\frac{k}{\pi a^{2} \varepsilon_{0}\left|\overline{E_{i n c}}\right|^{2}} \sum_{i=1}^{N} \operatorname{Im}\left(\bar{E}_{i n c, i}^{*} \cdot \bar{P}_{i}\right) \\
Q_{\text {sca }}=\frac{k^{4}}{6 \pi^{2} a^{2} \varepsilon_{0}^{2}\left|\overline{E_{i n c}}\right|^{2}} \sum_{i=1}^{N}\left|\bar{E}_{i n c, i}^{*} \cdot \bar{P}_{i}\right|^{2}
\end{gathered}
$$

and the absorption coefficient is $\mathrm{Q}_{\mathrm{abs}}=\mathrm{Q}_{\mathrm{ext}}-\mathrm{Q}_{\mathrm{sca}}$.

\subsection{Binary Particle Swarm Optimization Algorithm}

The PSO algorithm is an optimization procedure inspired by a colony such as birds which can improve its behaviors [20]. Any element of this colony is called a particle and moves in an n-dimensional space, correcting its trajectory based on the previous actions of itself and its neighboring particles. For each particle, velocity and displacement are updated based on the following relations [21]:

$$
\begin{aligned}
& v_{i}^{k+1}=w v_{i}^{k}+c_{1} r_{1}\left(p^{k}{ }_{i}-x_{i}^{k}\right)+c_{2} r_{2}\left(p_{g}^{k}-x_{i}^{k}\right) \\
& x_{i}^{k+1}=x_{i}^{k}+v_{i}^{k+1}, i=1, \ldots n
\end{aligned}
$$

where $k$ is the number of the current iteration, $n$ is the number of particles, $w$ is the inertia weight, $c_{1}$ and $c_{2}$ are acceleration parameters, and finally $r_{1}$ and $r_{2}$ are random parameters between 0 and 1 . The best position for the $i$-th particle which has been stored so far is represented as [22]:

$$
\mathbf{P}_{i}^{k}=\left[p_{1}^{k}, p_{i}^{k} \ldots \ldots \ldots \ldots, p_{n}^{k}\right]^{T}
$$

All the $\mathbf{P}_{i}^{k}$ (p_best) are evaluated by a fitness function. The best particle among all $p_{-}$best is represented as $p_{g}^{k}$ (Gbest_best_value), which in a minimization problem $p_{g}^{k}$ is the smallest member of the $\mathbf{P}_{i}^{k}$ vector while for a maximization problem it is the largest member of $\mathbf{P}_{i}^{k}$. PSO was designed for continuous problems, but cannot deal with 
discrete problems. A new version of PSO, called Binary PSO (BPSO), was introduced by Kennedy and Eberhart in 1997 and applied to discrete binary variables. After that, many optimization problems in various areas were solved by this method. The position in BPSO is represented by a binary vector and the velocity is still a floating-point vector; however, velocity is used to determine whether the probability changes from 0 to 1 or from 1 to 0 when the positions of particles are being updated. The equation for updating the positions is then replaced with:

$$
\begin{aligned}
& \operatorname{sigmoid}\left(v_{i d}^{k}\right)=\frac{1}{1+e^{\left(-v_{i d}^{k}\right)}} \\
& x_{i d}^{k}=\left\{\begin{array}{cc}
1, & \text { if rand }<\operatorname{sigmid}\left(v_{i d}^{k}\right) \\
0 & \text { otherwise }
\end{array}\right.
\end{aligned}
$$

In order to achieve maximum absorption, extinction, and scattering coefficient spectra, BPSO has been used to design a nonperiodic array of plasmonic nanospheres. The presence or absence of nanospheres could be controlled by a matrix with binary entries. As seen, BPSO is an algorithm that minimizes a cost function. To use the BPSO algorithm for maximizing the extinction coefficient spectrum, the algorithm should minimize the following function:

$$
\text { Cost Function }=-\sum_{i=300}^{600} Q_{\text {ext }}
$$

and for maximizing the absorption and scattering coefficient spectra:

$$
\begin{aligned}
& \text { Cost Function }=-\sum_{i=300}^{600} Q_{a b s} \\
& \text { Cost Function }=-\sum_{i=300}^{600} Q_{\text {sca }}
\end{aligned}
$$

where $\mathrm{i}$ is the wavelength number and Qext, Qabs, and Qsca are the extinction, absorption, and scattering coefficients respectively.

\section{NUMERICAL RESULTS}

The BCDA method is applied and the absorption spectrum is plotted for periodic and nonperiodic arrays of gold nanospheres with $1 \mathrm{~nm}$ diameter and $2 \mathrm{~nm}$ edgeto-edge separations in 1, 2, and 3 dimensions. Figure 4(b) shows the absorption spectrum as a function of wavelength in the range of 300-600 $\mathrm{nm}$ for a 1D nonperiodic array. The present simulations show that the absorption spectrum

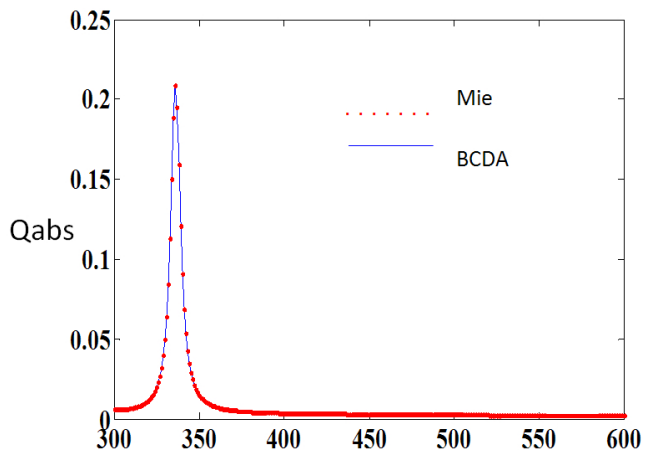

(a)

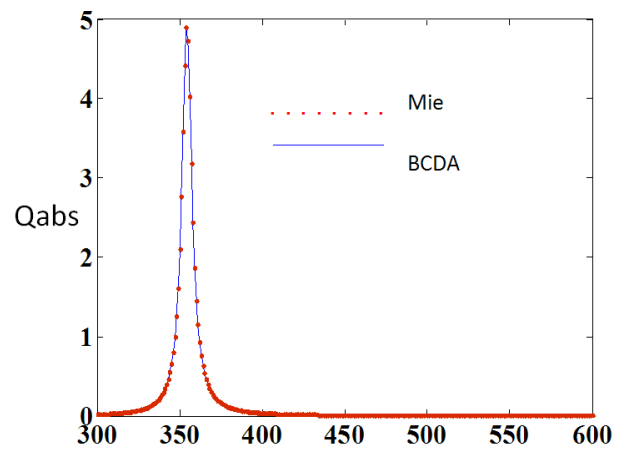

(b)

FIG. 4. (a) $\mathrm{Q}_{\mathrm{Abs}}$ for a $1 \mathrm{D}$ periodic array with $\mathrm{B}=\left[\begin{array}{lllll}1 & 1 & 1 & 1 & 1\end{array}\right]$.

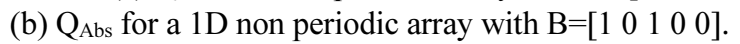

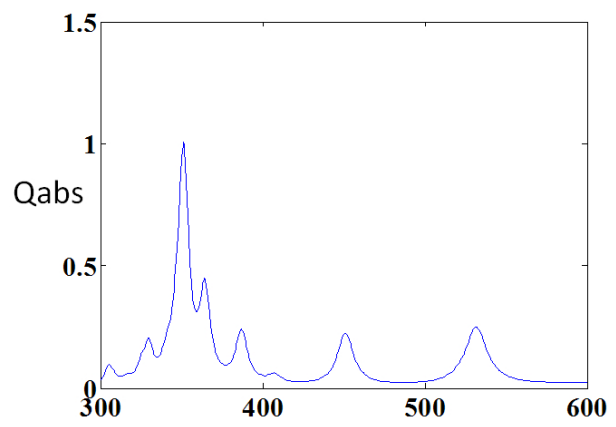

(a)

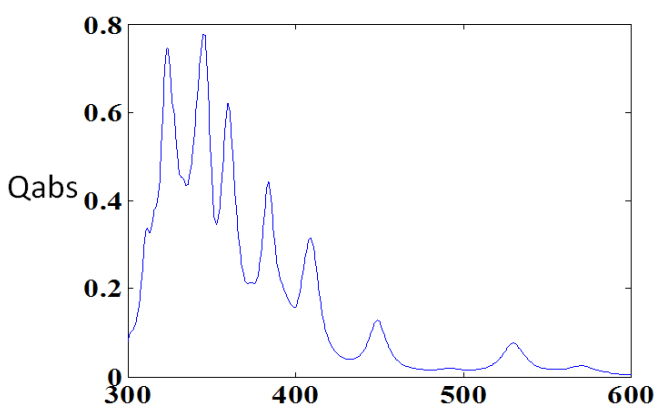

(b)

FIG. 5. (a) Absorption coefficient for a $2 \mathrm{D}$ nonperiodic structure with $\mathrm{B}=[01110 ; 01001 ; 10110 ; 01100 ; 01110]$, (b) Absorption coefficient for $3 \mathrm{D}$ nonperiodic structure with $\mathrm{B}=$ $[10001 ; 00010 ; 00100 ; 01011 ; 10100], \mathrm{B}(:,:, 2)=[10101 ; 0101$

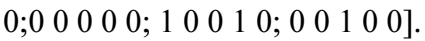


strongly depends on localized positions of nanospheres. On the other hand, further enhancement in absorption coefficient is possible for nonperiodic structure compare to the periodic array (Fig. 4(a)). These results are quite consistent

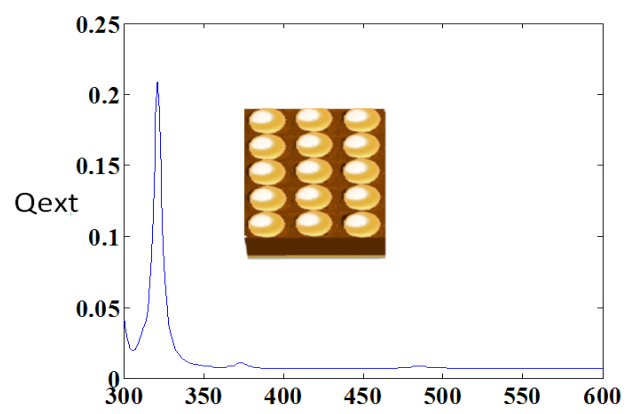

(a)

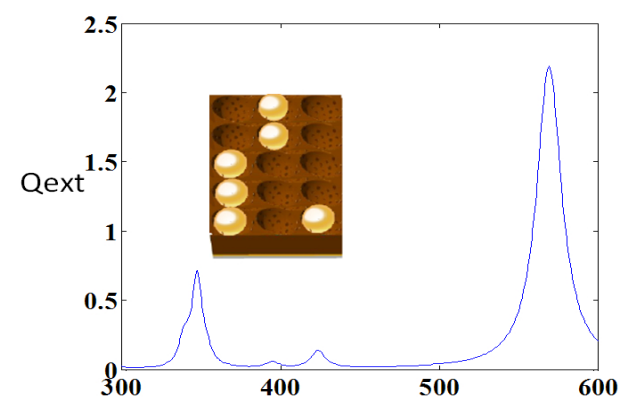

(b)

FIG. 6. (a) Extinction spectrum of a periodic 2D array with $3 * 5$ particles. (b) Extinction spectrum of nonperiodic $2 \mathrm{D}$ array

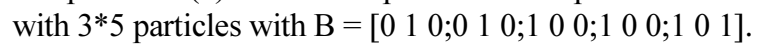

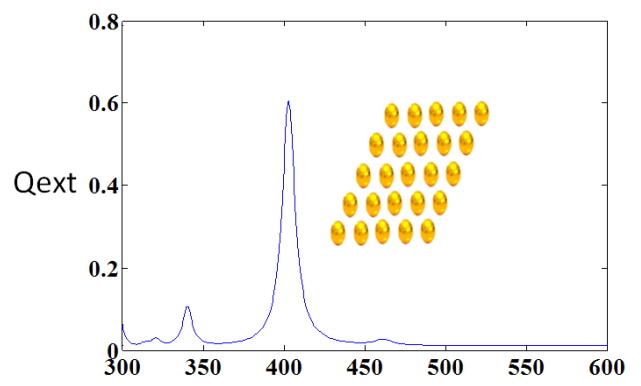

(a)

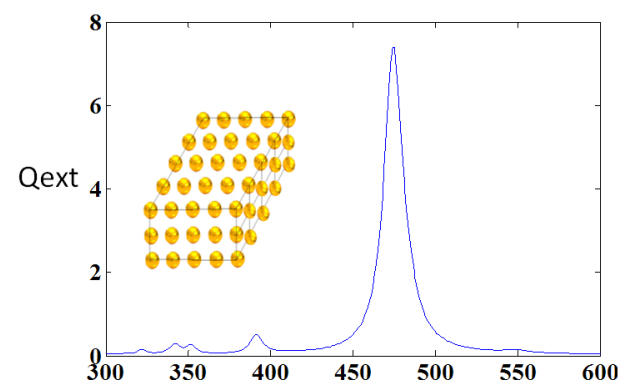

(c) with the results obtained using Mie theory [12]. Figure 5(a) shows this possibility for a 2D nonperiodic array. One benefit of increased material absorption coefficients is in solar cell. Then the effect of nonperiodic arrays of gold nanospheres on the absorption spectrum with a $3 \mathrm{D}$ nonperiodic array is investigated. Figure 5(b) shows the absorption spectrum as a function of wavelength for nonperiodic array in 3D coordinates. Figure 6(b) shows the extinction spectrum of a $2 \mathrm{D}$ nonperiodic array with $3 \times 5$ particles compared to that of periodic array (6(a)). The large extinction coefficient observed here was due to the interaction of metal nanoparticles via their strong near-field coupling. In this case the plasmon resonance of a nanoparticle acts as a light resonator and couples light to other resonators in the vicinity [23]. The BCDA method is applied and extinction coefficient spectra are plotted for 1, 2, 3, and 4 layers of gold nanospheres $(5 \times 5$ array) with $1 \mathrm{~nm}$ diameter and 2 $\mathrm{nm}$ edge-to-edge separations. Figure 7 shows these extinction spectra in the wavelength range of $300-600 \mathrm{~nm}$. Our simulations show that the extinction coefficient spectrum strongly depends on the number of layers of nanospheres, and further enhancement of the extinction coefficient is possible with multi periodic layers.

\section{OPTIMIZATION RESULTS}

Binary PSO and BCDA are used to optimize the farfield properties of transmitted light for optical applications. Our goal is to maximize Qext by optimizing 15 binary particles in $2 \mathrm{D}$ arrays $(3 \times 5)$. Figure $8($ a) shows the extinction

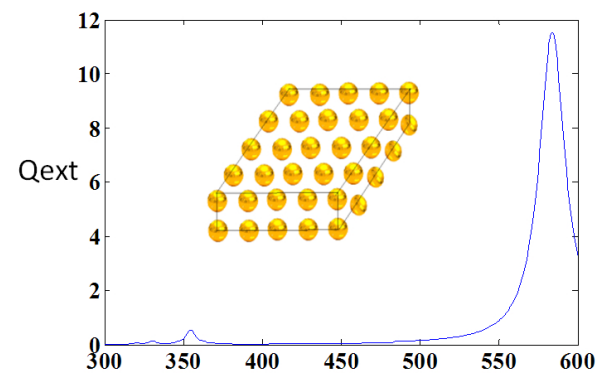

(b)

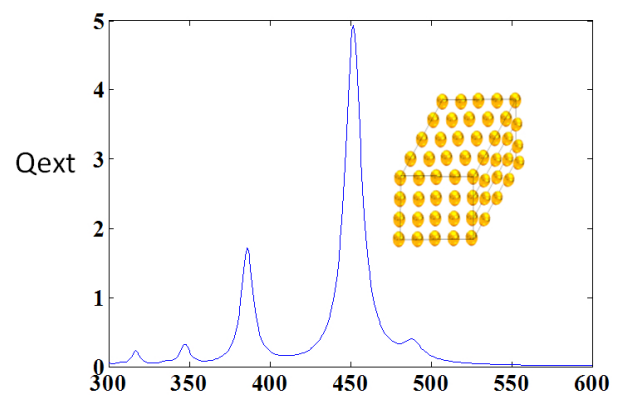

(d)

FIG. 7. Extinction coefficient spectra for (a) a 1-layer periodic array, (b) a 2-layer periodic array. (c) a 3-layer periodic array, and (d) a 4-layer periodic array. 


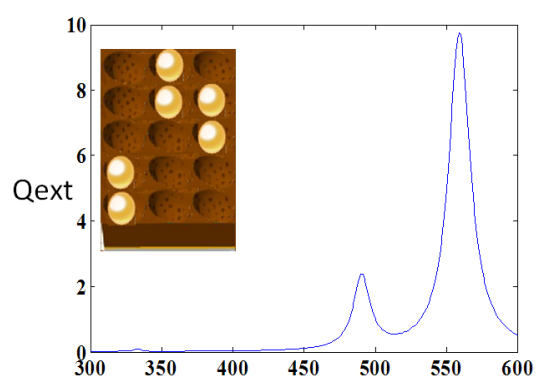

(a)

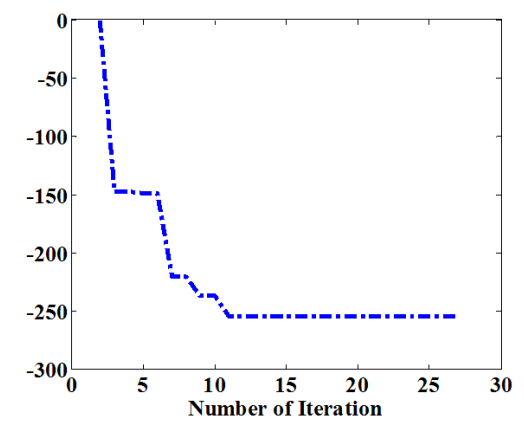

(b)

FIG. 8. (a) Extinction spectrum of the optimized array $(3 * 5)$

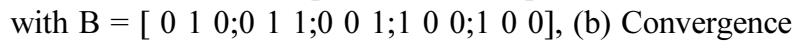
characteristic of BPSO.

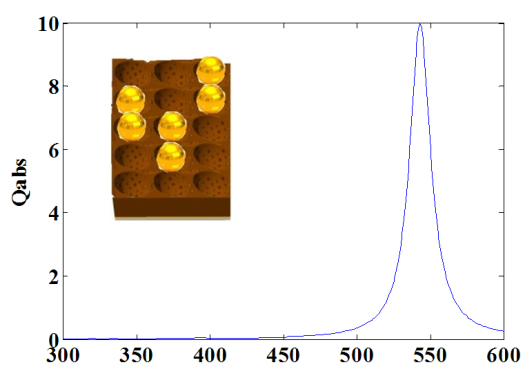

(a)

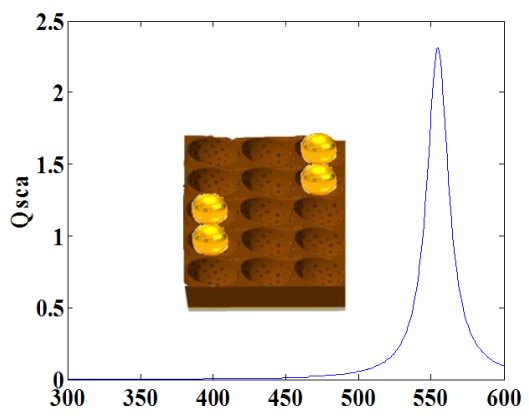

(b)

FIG. 9. (a) Absorption spectrum of the optimized array $(3 * 5)$

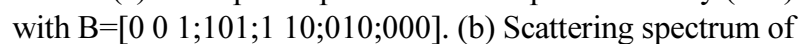
the optimized array $(3 * 5)$ with $\mathrm{B}=[001 ; 001 ; 100 ; 100 ; 000]$.

spectrum of the optimized array $(3 \times 5)$ compared to that of the periodic array (Fig. 6(a)). The maximum extinction in the entire range of $300-600 \mathrm{~nm}$ is 9.8 , which occurs at 560 $\mathrm{nm}$. The large extinction coefficient observed here is due to the interaction of metal nanoparticles via their strong near-field coupling. The convergence characteristic of the BPSO method is shown in Fig. 8(b). As seen, this algorithm had good performance in terms of convergence speed.

Next, the BPSO algorithm is applied to maximize absorption and scattering coefficients by optimizing the selection of binary particles. Figures 9(a) and (b) show the absorption and scattering spectra of the optimized array compared to those of the periodic array. One possible application of this method can be reduction of the scattering factor to have an optical invisibility cloak based on scattering cancellation. In addition to sensor, solar cell, and cloaking applications, BPSO can enhance the Raman scattering factor.

\section{CONCLUSION}

A numerical solution called the binary-coupled dipole approximation (BCDA) was described for simulating the effects of nonperiodic plasmonic nanoparticles on light properties. This method is useful for optimization problems to find the best nanoparticle array from all possible arrays. Finally, it was shown that using a nonperiodic structure could lead to higher extinction, absorption, and scattering coefficients. This approach can be useful in optical applications such as solar cells, bio sensor, and plasmonic nanoantennae.

\section{REFERENCES}

1. Y. Huang, X. Duan, Q. Wei, and C. M. Lieber, "Directed assembly of one-dimensional nanostructures into functional networks," Science 291, 630-633 (2001).

2. M. Law, D. J. Sirbuly, J. C. Johnson, J. Goldberger, R. J. Saykally, and P. Yang, "Nanoribbon waveguides for subwavelength photonics integration," Science 305, 1269-1273 (2004).

3. E. Katz and I. Willner, "Integrated nanoparticle-biomolecule hybrid systems: Synthesis, properties, and applications," Angewandte Chemie International Edition 19, 6042-108 (2004).

4. K. Kneipp, Y. Wang, H. Kneipp, L. T. Perelman, I. Itzkan, R. R. Dasari, and M. S. Feld, "Single molecule detection using surface-enhanced Raman scattering (SERS)," Phys. Rev. Lett. 78, 1667-1670 (1997).

5. J. S. Biteen, D. Pacifici, N. S. Lewis, and H. A. Atwater, "Enhanced radiative emission rate and quantum efficiency in coupled silicon nanocrystal-nanostructured gold emitters," Nano Lett. 9, 1768-1773 (2005).

6. K. R. Catchpole and A. Polman, "Plasmonic solar cell," Opt. Express 16, 21793-21800 (2008).

7. S. V. Boriskina, A. Gopinath, and L. D. Negro, "Optical gap formation and localization properties of optical modes in deterministic aperiodic photonic structures," Opt. Express 16, 18813-18826 (2008).

8. M. R. Hormozi-Nezhad, P. Karami, and H. Robatjazi, "A 
simple shape-controlled synthesis of gold nanoparticles using nonionic surfactants," An International Journal to Further the Chemical Sciences 3, 7726-7732 (2013).

9. M. L. Personick, M. R. Langille, J. Zhang, and C. A. Mirkin, "Shape control of gold nanoparticles by silver underpotential deposition," Nano Lett. 8, 3394-3398 (2011).

10. H. Wu, C. J. Choi, and B. T. Cunningham, "Plasmonic nanogap-enhanced Raman scattering using a resonant nanodome array," in Proc. Conference on Lasers and Electro-Optics (San Jose Convention Center, San Jose, CA, USA, 2012), CM3B.2.

11. G. O. Kawamura, M. Nogami, and A. Matsuda, "Shapecontrolled metal nanoparticles and their assemblies with optical functionalities," Journal of Nanomaterials 3, 1-17 (2013).

12. E. C. Le Ru and P. G. Etchegoin, Principles of Surface Enhanced Raman Spectroscopy and Related Plasmonic Effects (Elsevier Science, 2008).

13. L. D. Negro, N. N. Feng, and A. Gopinath, "Electromagnetic coupling and plasmon localization in deterministic aperiodic arrays," J. Opt. A, Pure Appl. Opt. 10, 064013 (2008).

14. L. Negro and N. Feng, "Spectral gaps and mode localization in Fibonacci chains of metal nanoparticles," Opt. Express 22, 14396-14403 (2007).

15. J. M. Luck, "Cantor spectra and scaling of gap widths in deterministic aperiodic systems," Phys. Rev. B 39, 58345849 (1989).
16. C. Forestiere, G. Miano, S. V. Boriskina, and L. Dal Negro, "The role of nanoparticle shapes and deterministic aperiodicity for the design of nanoplasmonic arrays," Opt. Express 17, 9648-9661 (2009).

17. E. M Purcell and C. R. Pennypacker, "Scattering and adsorption of light by nonspherical dielectric grains," Astrophys J. 186, 705-714 (1973).

18. B. T. Draine, "The discrete-dipole approximation and its application to interstellar graphite grains," Astrophys J. 333, 848-872 (1988).

19. C. F. Bohren and D. R. Huffman, Absorption and Scattering of Light by Small Particles (John Wily and Sons, 1998).

20. M. Bozorgi and N. Granpayeh, "Directional emission from photonic crystal waveguide output by terminating with CROW and employing the PSO algorithm," J. Opt. Soc. Korea 15, 187-195 (2011).

21. F. Emami and M. Akhlaghi, "Gain ripple decrement of S-band Raman amplifier," IEEE Photon. Technol. Lett. 24, 1349-1352 (2012).

22. M. Akhlaghi and F. Emami, "Fuzzy adaptive modified PSO-algorithm assisted to design of photonic crystal fiber Raman amplifier," J. Opt. Soc. Korea 17, 237-241 (2013).

23. Y. Nishijima, L. Rosa, and S. Juodkazis, "Long-range interaction of localized surface plasmons in periodic and random patterns of Au nanoparticles," J. Appl. Phys. A 113, November (2013). 